\title{
REPERCUSSÃO DA COVID-19 PARA A SAÚDE MENTAL E RISCO DE SUICÍDIO
}

\author{
Girliani Silva de Sousa' \\ ORCID: 0000-0002-0988-5744 \\ Natália de Magalhães Ribeiro Gomes" \\ ORCID: 0000-0003-1717-8692 \\ Tarcisia Castro Alves"' \\ ORCID: 0000-0002-7567-7636 \\ Maria Odete Pereira' \\ ORCID: 0000-0002-9418-2524
}

' Universidade Federal de São Paulo. São Paulo, São Paulo, Brasil.

" Universidade Federal de Minas Gerais. Belo Horizonte, Minas Gerais, Brasil.

I"' Universidade Federal da Bahia. Vitória da Conquista, Bahia, Brasil.

Autor Correspondente:

Girliani Silva de Souza E-mail: girlianis@gmail.com

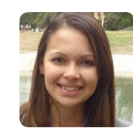

Como citar:

Sousa GS, Gomes NMR, Alves TC, Pereira MO. Repercussão da COVID-19 para a saúde mental e risco de suicídio. In: Esperidião E, Saidel MGB (Orgs.). Enfermagem em saúde mental e COVID-19. 2.ed.rev. Brasília, DF: Editora ABEn; 2020. p. 56-64. (Série Enfermagem e Pandemias, 4). https://doi.org/10.51234/aben.20.e04.c08

\section{INTRODUÇÃO}

A Covid-19, termo derivado do inglês coronavirus disease 2019, causada pelo vírus Sars-CoV-2, da família Coronaviridae, popularmente conhecido como novo coronavírus e causador da Síndrome Respiratória Aguda, teve sua primeira ocorrência notificada em dezembro de 2019, em Wuhan, província de Hubei na China e se disseminou rapidamente pelo mundo, inclusive no Brasil ${ }^{(1)}$.

O primeiro caso da Covid-19, no Brasil, foi detectado na cidade de São Paulo. Com uma população de 22 milhões, a doença logo se espalhou pela região metropolitana e, concomitantemente, pelo Brasil, com mais de 211 milhões de habitantes. Assim, em noventa dias contabilizou-se 56.109 mortes no País e 1.280.054 de casos confirmados ${ }^{(2)}$.

À medida que os casos de Covid-19 foram e são anunciados, as pessoas vêm sofrendo com sentimentos de angústia e de estresse frente ao insuficiente conhecimento científico acerca da doença, a sua alta velocidade de disseminação, risco iminente de infecção, a capacidade de provocar mortes em populações vulneráveis e a incerteza econômica, especialmente em países de baixa e média renda, como o Brasil, onde normalmente existem medidas limitadas de proteção social ${ }^{(3)}$. Nesse panorama, pessoas que possuem comorbidades, como doenças crônicas, terminais e transtornos mentais estão mais expostas a terem repercussões da Covid-19 em sua saúde mental(3). Esses dados são confirmados a partir de evidências iniciais relatadas acerca da Covid-19 na China e em outros países sinalizam para o aumento de problemas de saúde mental na população em geral e em grupos vulneráveis, com as medidas restritivas de isolamento social para a prevenção à contaminação da doença(4).

O Brasil se destaca como um dos países com o maior número de casos de infectados e de mortos pela Covid-19(5). Essa situação é ainda mais desafiadora devido ao contexto de elevada desigualdade social, com populações vivendo 
em condições precárias de habitação, saneamento, desemprego e aumento da pobreza extrema, que levam as pessoas a terem empregos informais e a viverem em situação de aglomeração.

Durante o período de pandemia no Brasil, a adoção de diferentes estratégias de isolamento social, vertical ou horizontal ainda são limitados e insuficientes. Isso se dá, em parte, pela crise da dimensão ética-econômica-fiscal-política-institucional, na qual o governo minimiza a gravidade do problema, diminuindo seus impactos na vida da população sem políticas públicas efetivas. Com isso, instala-se um processo burocrático para a concessão de auxílio-emergencial para a população economicamente vulnerável, o que leva à morosidade, com consequente aglomeração de pessoas em filas bancárias, com maior exposição ao vírus e contágio. A falta de renda mínima para suprir as necessidades básicas, como alimentação e habitação, impacta, sobremaneira, na qualidade de vida desse segmento populacional.

Em adição, a dificuldade de monitoramento e vigilância de casos e contatos diretos, a fragilidade do Sistema de Saúde para testagem e identificação precoce dos infectados e a falta de leitos e de respiradores em Unidades de Terapia Intensiva (UTI) levam as pessoas a sentirem-se desamparadas pelo Estado.

As condições precárias de vida, somada às questões políticas, econômicas, sociais e psicológicas relacionadas ao isolamento social, inevitavelmente, geram sofrimento psíquico em muitos e levam tantos outros, que já vivenciam um processo crônico de adoecimento psíquico, a se desestabilizarem. Salienta-se que a ansiedade, o medo do desconhecido, o desespero e a desesperança estão associados a maior risco de suicídio.

As publicações científicas evidenciam que a medida em que a pandemia avança é premente a preocupação quanto ao aumento do suicídio, levado pelo impacto dos efeitos econômicos, pela ocorrência de fatores precipitantes, como a violência doméstica e o consumo de álcool e outras drogas, assim como, pela possibilidade de ampliação dos riscos de suicídio trazidos pelo estigma para com os indivíduos com Covid-19 e as suas famílias. Tais efeitos serão sentidos não apenas pela população em geral, assim como por aqueles com maior exposição a doenças provocados pela Covid-19, como os trabalhadores da linha de frente. Nessa perspectiva, faz-se a necessário o desenvolvimento de estratégias de prevenção, acompanhamento e posvenção do suicídio(6-7).

\section{OBJETIVO}

Refletir estratégias de cuidado à pessoa com comportamento suicida no contexto da pandemia da Covid-19 no Brasil.

\section{MÉTODOS}

Trata-se de um estudo de cunho teórico-reflexivo embasado em publicações científicas disponíveis e em literatura que aborda o comportamento suicida.

Os autores realizaram uma busca não sistematizada nas bases de dados PUBMED (PMC) e no Scielo no mês de junho de 2020 com os descritores de saúde: pandemia, COVID-19, saúde mental e risco de suicídio. Adicionalmente, incluiu-se livros teóricos sobre suicidologia para subsidiar as reflexões teóricas para a pandemia na sociedade contemporânea.

A partir da leitura crítica dos artigos encontrados e dos livros clássicos sobre o tema, essa reflexão foi dividida nos três eixos, sendo estes: reflexão acerca do suicídio no cenários da covid-19; a garantia de necessidades que garantam a vida e boas práticas em saúde para a prevenção ou cuidado de suicídio em tempos de covid-19.

\section{REFLEXÃO ACERCA DO SUICÍDIO NO CENÁRIO DA COVID-19}

O tema do suicídio se coloca em evidência, preocupando ainda mais os estudiosos da área de saúde mental durante a pandemia de Covid-19, pelo risco que oferece à vida, pelas restrições decorrentes do isolamento 
social, pelas incertezas relacionadas à assistência à saúde necessária em condição de agravamento clínico, pela morte de familiares e amigos, geradas por ela, bem como pela falta de perspectiva e vulnerabilidade, resultantes da crise econômica, vivenciada por milhões de pessoas.

Em complementação, estes impactos são intensificados pela crise política neoliberal vivenciada pela população brasileira, que não reconhece o Estado como promotor do bem-estar social, na garantia de direitos sociais mínimos dos cidadãos, para diminuir os efeitos deletérios da pandemia na maior e pior crise econômica que o mundo se depara, desde a primeira guerra mundial. Em decorrência disto, um terço da população resiste ao isolamento social e à paralisação dos serviços não essenciais, em defesa e manutenção de vida humanas.

Neste cenário de agravos, as pessoas com transtornos mentais, associados ou não ao uso problemático de álcool e outras drogas, outras morbidades e comorbidades, apresentam ainda maior vulnerabilidade para o suicídio ${ }^{(4)}$. A explicação para essa associação se deve ao aumento da ansiedade e do distanciamento social, que faz com que as pessoas estejam reclusas em suas casas há no mínimo quatro meses, como no caso brasileiro. No tocante à neurociências, a exposição e o acompanhamento do número expressivo de casos de infectados e de morte por Covid-19, acarreta no estado hipervigilante estendido que pode ativar o eixo hipotálamo-hipófise-adrenal e causar níveis elevados de glicocorticóides. Isso, por sua vez, está relacionado a transtornos de ansiedade e depressão(3)

No entanto, não se pode ignorar que além das mortes pela Covid-19, que causam comoção coletiva, há pessoas que lidam melhor com sua dor, tristeza ou angústia e, por isso, podem desejar deixar de viver ${ }^{(8)}$.

Émile Durkheim ${ }^{(9)}$ ao realizar um estudo teórico a respeito do suicídio, dentro dos pressupostos sociológicos, trouxe à tona que o fenômeno é complexo e influenciado por diversos aspectos de ordem individual e coletiva ${ }^{(10)}$. Para este autor, o suicídio deve ser compreendido pelo olhar sociológico, frente aos fatores sociais e, não apenas pelas motivações pessoais envolvidas nos atos de destruição.

O suicídio é compreendido como toda morte que resulta, mediata ou imediatamente, de um ato, realizado pela própria vítima e que ela sabia que produziria esse resultado. O suicídio é, antes de tudo, o ato de desespero de uma pessoa que não faz mais questão viver ${ }^{(9)}$.

Para Durkheim ${ }^{(9)}$, estudar o conjunto dos suicídios cometidos em uma determinada sociedade, durante um período específico de tempo, permitiu identificar que crises contemporâneas afetam temporariamente a situação social, como o ocorrido em intensas crises econômicas, como por exemplo, a crise de 1848, em que se observou aumento de mortes por suicídio em todos os Países europeus. Quando estas crises se tornam crônicas, causam modificações sociais bruscas e progressivas. Desta forma, a ruptura do equilíbrio social, quando eclode repentinamente, demanda um tempo para produzir todas as suas consequências.

Isto quer dizer que, se atualmente, o debate está em torno de como controlar o contágio e conter o número de mortes pela Covid-19; com a necessidade de aumentar o número de leitos de internações em UTI; na aquisição de maior número de respiradores; com a implantação de leitos em hospitais de campanha e a adoção de medidas protetivas e restritivas de combate ao novo coronavírus, nos próximos meses ou anos, a preocupação social se voltará para o aumento no número de casos de suicídio.

No Brasil, as ações preventivas para o suicídio eram e continuam sendo incipientes e insuficientes. Apesar de existirem normativas e regulamentos, tais como a Portaria $n^{\circ} 1.876$ de $2006^{(10)}$, que instituiu as Diretrizes Nacionais para Prevenção do Suicídio e a Portaria no 1271 de $2014^{(11)}$, que dá direcionamento para a notificação compulsória, da tentativa de suicídio e da automutilação, na prática, o desafio reside em acolher esses indivíduos e oferecer tratamento adequado e afetivo, nos serviços de saúde e em tempos de pandemia.

Do ponto de vista social, alguns países desenvolveram estratégias efetivas de cuidado em saúde mental, com o monitoramento das pessoas por meio de teleassistência, entretanto, no contexto brasileiro, de elevada desigualdade social, muitos não têm acesso à internet ou este é limitado, dificultando as chamadas de vídeo ou a participação de grupos virtuais. Nestes contextos, prevalecem o acompanhamento por telefone, mas nem sempre as pessoas tem em sua casa um ambiente seguro e acolhedor, onde possam verbalizar seus pensamentos, afetos e sentimentos ${ }^{(13)}$. 
Do ponto de vista econômico, o fechamento do comércio e dos serviços não essenciais, como salões de beleza e academias, entre outras modalidades, culminou na suspensão salarial dos trabalhadores e na dependência do auxílio emergencial, pago pelo Governo Federal às famílias. Além disso, estabelecimentos menores, sem perspectiva de retorno, tiveram suas atividades encerradas, com demissão dos funcionários. Enfatiza-se que, anteriormente à pandemia, o Brasil já enfrentava uma crise econômica com déficit do produto interno bruno (PIB), o que fez com que mais de 38 milhões de pessoas migrassem para o trabalho, sem garantias trabalhistas e previdenciárias.

Durkheim ${ }^{(9)}$, afirma que quando a sociedade se sente desamparada frente a uma crise econômica que afeta sua segurança e bem-estar, observa-se maior vulnerabilidade para o suicídio. Neste contexto, a vida se torna mais difícil devido à ruptura de equilíbrio no suprimento de suas necessidades, causando dor e sofrimento, com a satisfação pela vida diminuída ou até menos aniquilada.

Nesta perspectiva, Shneidman ${ }^{(12)}$, ao propor o método de autópsia psicológica como estratégia para compreender o sofrimento do outro e a morte pelo suicídio, desvela que o suicídio possui um entrelaçamento de circunstâncias sociais e de fatores psicológicos que irão influenciar no desejo de morrer. Assim, Shneidman ${ }^{(12)}$ define o suicídio como uma dor psicológica extrema por não se ter as necessidades humanas supridas, havendo um esvaziamento de forças psíquicas para viver.

As reflexões propostas por Durkheim se articulam com a sociedade contemporânea no tocante a vivência e as repercussões psicossociais advindas pela pandemia por COVID-19. Acredita-se que a pandemia por COVID-19 intensificou uma desordem econômica-social anteriormente vivenciada em nível mundial e trouxe influências nas vidas dos indivíduos em nível global, pelo alcance que teve a doença e pela velocidade com a qual se disseminou. Por conseguinte, o isolamento social como única medida protetiva viável para conter a infecção pode potencializar conflitos familiares, acarretar em sintomas ansiosos, depressivos e o desejo de morrer por não ter estratégias eficazes de enfrentamento para garantir o suprimento das necessidades básicas de vida e o cuidado de si. O desejo de morrer pode aumentar em pessoas que se encontravam anteriormente a pandemia por COVID-19 em sofrimento psíquico, com problemas de saúde crônica, conflitos familiares e dificuldades financeiras.

\section{A GARANTIA DE NECESSIDADES BÁSICAS QUE PROMOVAM A VIDA}

Maslow $^{(14)}$, argumenta que as necessidades humanas correspondem às fisiológicas, de segurança, sociais, status, estima e autorrealização. Todas estão interligadas e exercem influência na percepção de saúde mental das pessoas. Nesse contexto, o cuidado de si é uma estratégia para ter controle de sua vida pessoal. O cuidado de si pode ser caracterizado como uma elaboração do sujeito por meio de uma transformação subjetiva e da consequente constituição de novos modos de vida, que se manifestam, pelos atos e condutas e por um conjunto de práticas e técnicas que são exercidas de si para consigo mesmo. Para isso, assegurar a garantia das necessidades humanas pode ser um caminho para a promoção da saúde mental (15).

Quando as necessidades humanas não são supridas, sobrevêm sentimentos negativos como: angústia; melancolia; tristeza; frustração; desesperança; baixa autoestima; pessimismo e insegurança. Esses sentimentos agem na vida do ser humano de forma que busquem recompensas para minimizar o sofrimento psíquico, como pensamentos persistentes; choro constante; falta de energia para realizar as atividades diárias e muitas vezes, $\mathrm{O}$ emprego de dispositivos ineficazes como o uso abusivo de substâncias psicoativas e medicalização excessiva ${ }^{(14)}$.

No contexto da pandemia de Covid-19, as medidas preventivas, principalmente no tocante ao distanciamento e isolamento social, repercutem em todas as necessidades humanas, especialmente no tocante às necessidades sociais. $\mathrm{O}$ isolamento social durante a Pandemia levou os seres humanos a buscarem novas formas de contato, a fim continuarem interagindo entre si. Mesmo assim, essa realidade repercute negativamente nas necessidades de status, estima e de autorrealização, como autoconfiança e reconhecimento, entre outras ${ }^{(14)}$. 
Em adição, o estigma, o preconceito e o despreparo de alguns profissionais na Atenção Primária à Saúde, constituem verdadeiros desafios para a identificação do risco de suicídio, pois a restrição à visita domiciliar, pelo agente comunitário de saúde e profissionais da saúde, e as condições precárias de habitação, que colocam as famílias em situação de aglomeração nos lares, somados aos efeitos psíquicos do isolamento social, distanciamento social como medidas preventivas e quarentena, para quem está infectado, afetam as necessidades básicas dessas pessoas e faz com que o risco para o suicídio se mantenha na invisibilidade, até mesmo no ambiente doméstico ${ }^{(16)}$.

A garantia de seguridade social está intimamente ligada aos recursos financeiros que permitirão ao ser humano suprir outras necessidades, mas o panorama brasileiro é de elevação dos índices de pobreza nos cinco últimos anos. Segundo dados da Pesquisa Nacional por Amostra de Domicílios- PNAD, em 2019 cerca de 170 mil de pessoas passaram para a extrema pobreza ${ }^{(17)}$.

Em complementação, no primeiro semestre de 2020, de acordo com a PNAD, mais da metade da população brasileira está desempregada. Em decorrência da pandemia de Covid-19, a taxa de desemprego segue crescendo no país e atingiu 12,9\% no trimestre encerrado em maio. Assim sendo, o total de desempregados foi estimado em 12,710 milhões, 368 mil (3\%) a mais que no trimestre anterior e a perda de salário chegou perto de R\$ 11 bilhões em um único trimestre ${ }^{(17)}$.

Frente a este cenário, que não dispõem de mecanismos eficazes de superação a curto prazo, a ideia do suicídio surge como um desfecho para uma história existencial marcada pelo sofrimento e falta de perspectivas. Assim, o suicídio, portanto, pode ser compreendido como a impossibilidade de vislumbrar alternativas viáveis para a solução de seus conflitos, sendo a morte a única resposta viável para se ver livre da dor, angústia e sentimento de desvalia(4).

Lidar com o "suicídio" e possível aumento de prevalência, em decorrência da Covid-19 no Brasil, implica no aprendizado com o desespero humano, as imprevisibilidades da vida e com indivíduos que não exprimem desejos para se manterem vivos, pois perderam a esperança e fé na vida e, por outro lado, tem-se as estruturas de níveis macrossociais, que não conseguem suprir as necessidades do ser humano ${ }^{(7)}$.

Neste sentido, uma abordagem fenomenológica, como inspiração terapêutica, é também uma perspectiva para compreender o suicídio em tempos pandêmicos, para entender como o indivíduo vivencia seu sofrimento. A narrativa deste pode indicar os sentimentos, faltas e lacunas que rondam a existência do ser humano e as razões que este elenca para desistir de tudo, inclusive de sua vida. Compreender as condutas, atitudes e pensamentos adotadas pelo ser humano é um caminho paradigmático para reconhecer os sintomas manifestados pelo indivíduo e para entender como eles se manifestam nos contextos de vida tão singulares e individuais para cada pessoa. Dessa forma, é possível desenvolver boas práticas individualizadas em saúde mental para a prevenção ou cuidado de suicídio.

\section{BOAS PRÁTICAS EM SAÚDE PARA A PREVENÇÃO OU CUIDADO DE SUICÍDIO EM TEMPOS DE COVID-19}

Dentre os profissionais que compõem a equipe multiprofissional dos diversos serviços de saúde, destaca-se o enfermeiro. Assim, faz-se necessário que o enfermeiro desenvolva empatia ao sofrimento humano, ou seja, que o profissional se disponibilize a aproximar do usuário e estabelecer o relacionamento interpessoal ${ }^{(18)}$.

Por meio do relacionamento interprofissional o enfermeiro terá a possibilidade de conhecer as tramas pessoais, as vivências e os sentimentos que se aglutinam em situações de sofrimento intenso.

O relacionamento terapêutico se estabelece pela escuta terapêutica, com a pessoa com Covid-19 e familiares, que deve ser realizada já na admissão para internação, com o objetivo de reconhecer as potencialidades e ampliar os modos de enfrentamento do sofrimento vivenciado(19).

O cuidado somente é efetivo e real quando o enfermeiro considera os aspectos emocionais e psicológicos da pessoa que está adoecida e por seus familiares que sofrem com a perspectiva do óbito dado à gravidade da doença. Lidar com a morte nos remete a nossa própria finitude que atormenta e ameaça. 
Aos familiares que se veem frente à impossibilidade de visitas hospitalares, a videochamada é um recurso muito potente, bem como para a população em condição de isolamento social, pois em ambas as situações contribui sobremaneira para diminuir a angústia, saudade e/ou solidão.

A interação por videochamada é uma alternativa muito viável, pois pode ser realizada por meio de celular, que comum a maioria da população. O recurso ajuda a minimizar o sofrimento dos familiares e pessoa adoecida, causado pelas incertezas do tratamento e da evolução da Covid-19(13). Ademais, é uma forma de estabelecer uma relação terapêutica que prima pelo cuidado à subjetividade das pessoas e supera o cuidado prescritivo moldado apenas por técnicas e procedimentos.

Em serviços de Atenção Primária e especializados em saúde mental, o funcionamento do acolhimento é imprescindível para a identificação precoce e o manejo adequado das pessoas em risco de suicídio ${ }^{(16)}$. Em um primeiro momento, perguntar e explorar faz com que se identifique a intensidade da presença da ideação suicida e elaboração de plano suicida: Quais são as pistas verbais que a pessoa verbaliza? A pessoa pensa em se matar? Sente que a vida está tão difícil que vislumbra a morte como solução para seus problemas? Há planejamento do ato? A pessoa pensou em datas? Escreveu cartas de despedidas?

Por meio do acolhimento é possível que o enfermeiro, juntamente com o usuário, o leve a refletir acerca dos sentimentos; angústias; lacunas e circunstâncias que envolvem o desejo da de morrer.

Explorar os sentimentos é compreender o sentido do suicídio para pessoa e para que possa surgir oportunidades de reflexão acerca das possibilidades existenciais, ou seja, em quais momentos ela encontra sentido para sua existência. Nesse momento, o enfermeiro pode firmar um contrato terapêutico e verbalizar sua preocupação e intenção de orientar a pessoa em questão e, juntos, pensarem em formas de cuidado.

Além disso, é imprescindível incluir a família e rede extensa, para garantir suporte emocional para a pessoa em risco de suicídio. Em casos mais graves, em que a ideação suicida é persistente, há a necessidade de direcionamento para o acolhimento integral em Centros de Atenção Psicossocial (CAPS), com avaliação psiquiátrica para traçar o projeto terapêutico singular, com metas e estratégias de cuidado elaborados e equipe interprofissional do serviço, em corresponsabilização com a pessoa em sofrimento e família.

Outras formas de cuidado é por meio da teleassistência, que foi normatizada pela resolução do Cofen $\mathrm{n}^{\circ}$ 634 de $2020^{(20)}$, em que enfermeiros também podem monitor pessoas que possuem transtornos mentais, que é o grupo com maior vulnerabilidade para o suicídio e, com o apoio dos agentes comunitários de saúde, possam identificar pessoas que perderam seus empregos, vivem situações de violência doméstica e estão em sofrimento psíquico.

Por conseguinte, garantir o teleatendimento do profissional ou equipe de saúde que acompanha as pessoas em sofrimento psíquico é uma forma de assegurar o acompanhamento terapêutico e até mesmo o tratamento psiquiátrico, se corresponsabilizando com as mesmas, para que não se sintam abandonadas e abandonem o tratamento em curso.

Em complementação, ressalta-se que a terapêutica farmacológica deve ser assegurada e supervisionada, indiretamente, pela equipe interdisciplinar do serviço de saúde, em corresponsabilização com a família, de forma que um dos familiares supervisione diretamente. No entanto, se a pessoa residir sozinha ou estiver em situação de rua, essa supervisão da medicação deverá ser direta, por meio do agente comunitário, em visita domiciliar ou pessoa se dirigindo à unidade de saúde. Destaca-se que se houver necessidade, atendimentos e consultas presenciais devem ser oferecidas aos usuários, no serviço, durante a pandemia.

Amigos e familiares poderão também identificar alterações de comportamento e verbalizações de conteúdo anedônico e niilista e encorajar a pessoa a buscar ajuda profissional e serviço de saúde do Sistema Único de Saúde - SUS ou até mesmo tomar a iniciativa.

Outros mecanismos de enfrentamento podem ser encorajados, como a prática de exercícios físicos; meditação; promoção à espiritualidade; prática de atividades que promovam o prazer e autoestima/autoimagem como: cozinhar; trabalhos manuais e artísticos; danças e práticas complementares, entre outras. 
Cabe salientar que a divulgação de campanhas nas mídias, que busquem promover a saúde mental é um caminho para combater o estigma e o tabu acerca do suicídio, haja vista que as pessoas ainda se envergonham e não se admitem expressar tristeza, solidão, angústias e sentimentos de menos valia, por receio de serem julgadas e criticadas.

Assim sendo, na prevenção do suicídio, a apresentação midiática de notícias e informações devem ocorrer na perspectiva da educação em saúde, para a população em geral, reconhecendo os fatores de risco e de proteção e, assim, desconstruir mitos e a desinformação a respeito do tema.

Salienta-se que apesar de se dar ênfase ao trabalho do enfermeiro, neste texto, todas as recomendações aplicam-se às demais formações profissionais que atuam na área da saúde, que compõem as equipe interdisciplinar e interprofissional, haja vista que estes agregam conhecimentos e esforços para os cuidados da pessoa que que não vê mais sentido na vida e no seu viver. Afinal, não é recomendável que se realize um trabalho solitário no cuidado à pessoa com comportamento suicida.

Portanto, a disponibilidade afetiva e empatia cabem a todos os profissionais de saúde, que devem acolher com solidariedade e compaixão o sofrimento alheio, ocasionado pelas adversidades que a vida lhe impõe. A afetividade não é garantia de que a pessoa não tentará o suicídio, mas expressa a sensibilidade e habilidade de que a pessoa em questão está sendo compreendida. $O$ ato de escutar sempre passa ao outro a ideia de estar sendo acolhido, acalentado, de ser compreendido, no seu desespero existencial.

Espera-se que essas reflexões possam contribuir para a sensibilização dos profissionais de saúde para terem uma prática empática, humanizada e que valorize a subjetividade do ser humano.

\section{Limitações do Estudo}

Por se tratar de um estudo teórico-reflexivo, as autoras consideram essencial a realização de estudos quantitativos e qualitativos que identifiquem os fatores de risco para o suicídio e relatem as experiências de pessoas com risco para suicídio. Estudos desta natureza darão visibilidade aos elementos inerentes ao fenômeno vivenciado durante a pandemia de Covid-19.

\section{Contribuições para a Área}

O estudo apresentou várias boas práticas, tais como as discutidas por Gambarelli ${ }^{(18)}$ e Unicovsky ${ }^{(19)}$, de que a Enfermagem e demais profissionais de saúde podem e devem lançar mão para a promoção à vida e prevenção de suicídio.

Além disso, este estudo se pautou em ressaltar os fatores de risco comportamentais, psicológicos e sociais para o suicídio na pandemia da covid-19 amparado nos referenciais teóricos da suicidologia e nos recentes estudos sobre o impacto da pandemia na saúde da população. A partir disso, fazemos uma reflexão sobre como as boas práticas de enfermagem podem atuar na prevenção do ato fatal.

\section{CONSIDERAÇÕES FINAIS}

Sabe-se que uma das repercussões durante e após pandemia será percebida no aumento nas taxas de suicídio, comportamentos suicidas, automutilação, uso abusivo de substâncias psicoativas e transtornos mentais.

Neste sentido, refletir acerca do suicídio é analisar o fenômeno em busca de respostas em conjunto com a pessoa que pensa em acabar com sua própria existência para criar estratégias que visem aliviar a sua dor psíquica.

A Enfermagem e demais profissionais de saúde precisam empregar a escuta, o acolhimento, o relacionamento interpessoal, a teleassistência e outras estratégias criativas para o monitoramento e acompanhamento das pessoas com risco para o suicídio. 
Nesta perspectiva, a articulação com a família e rede social significativa é essencial para que os laços sociais se fortaleçam e os recursos para lidar com os momentos de desespero e impulsividade possam ser reconhecidos e utilizados pela pessoa.

\section{AGRADECIMENTO}

Ao Departamento de Enfermagem Psiquiátrica e Saúde Mental/Associação Brasileira de Enfermagem (DEPSM/ABEn).

\section{REFERÊNCIAS}

1. Ahmed Z, Ahmed O, Aibaoa Z, Hanbina S, Siyuc L, Ahmad A. Epidemic of COVID-19 in China and associated Psychological Problems. Asian Journal of Psychiatry. 2020; 51. doi.org/10.1016/j.ajp.2020.102092

2. Ministério da Saúde. (Brasil). Painel de casos de doença pelo coronavírus 2019 (COVID-19) no Brasil pelo Ministério da Saúde [internet]. Ministério da Saude; 2020. [acesso 2020 Jul 17]. Disponivel em: https://covid.saude.gov.br/

3. Castro-de-Araujo LFS, Machado DB. Impact of COVID-19 on mental health in a Low and Middle-Income Country. Ciênc. saúde coletiva. 2020;25(1): 2457-2460. doi.org/10.1590/1413-81232020256.1.10932020.

4. Holmes EA, O'Connor RC, Perry VH, Tracey I, Wessely S, Arseneault L, Ballard C, Christensen H, Cohen Silver R, Everall I, Ford T, John A, Kabir T, King K, Madan I, Michie S, Przybylski AK, Shafran R, Sweeney A, Worthman CM, Yardley L, Cowan K, Cope C, Hotopf M, Bullmore E. Multidisciplinary research priorities for the COVID-19 pandemic: a call for action for mental health science. Lancet Psychiatry. 2020 Jun;7(6):547-560. doi: 10.1016/S2215-0366(20)30168-1.

5. Organização Pan Americana (OPAS). Folha informativa - COVID-19 (doença causada pelo novo coronavírus). Organização Pan Americana; 2020. [acesso 2020 Jul 17]. Disponível em: https://www.paho.org/bra/index.php?option=com_content\&vi ew=article\&id=6101:covid19\&ltemid $=875$

6. Gunnell D, Appleby L, Arensman E, Hawton K, John A, Kapur N, et al. Suicide risk and prevention during the Covid-19 pandemic. The Lancet Psychiatry. 2020. doi.org/10.1016/S2215-0366(20)30171-1.

7. Fiocruz. Saúde mental e atenção psicossocial na Pandemia Covid-19. Suicídio na pandemia Covid-19. Fiocruz; 2020. [acesso 2020 Jul 17]. Disponível em:https://www.fiocruzbrasilia.fiocruz.br/wp-content/uploads/2020/05/cartilha_prevencaosuicidio.pdf

8. Razai Mohammad S, Oakeshott Pippa, Kankam Hadyn, Galea Sandro, Stokes-Lampard Helen. Mitigating the psychological effects of social isolation during the covid-19 pandemic. BMJ. 2020; 369:m1904. doi.org/10.1136/bmj.m1904

9. Durkheim E. O Suicídio. São Paulo: Martin Claret; 2002.

10. Brasil. Ministério da Saúde. Portaria no 1.876, de 14 de agosto de 2006. Institui Diretrizes Nacionais para Prevenção do Suicídio, a ser implantadas em todas as unidades federadas, respeitadas as competências das três esferas de gestão. Diário Oficial da República Federativa do Brasil, Brasília (DF), 2006.

11. Brasil. Ministério da Saúde. Portaria n 1.271, de 6 de junho de 2014. Define a Lista Nacional de Notificação Compulsória de doenças, agravos e eventos de saúde pública nos serviços de saúde públicos e privados em todo o território nacional, nos termos do anexo, e dá outras providências. Diário Oficial da República Federativa do Brasil, Brasília (DF), 2014

12. Shneidman E. Suicide as psychache: A clinical approach to self-destructive behavior. New Jersey, NJ: Jason Aronson; 2004.

13. Caetano R, Silva AB, Guedes ACCM, Paiva CCND, Ribeiro GDR, Santos DL, Silva RMD. Challenges and opportunities for telehealth during the COVID-19 pandemic: ideas on spaces and initiatives in the Brazilian context. Cadernos de Saúde Pública. 2020;36: e00088920.

14. Maslow AH. Motivación y personalidad. Barcelona: Ed. Sagitário; 1954.

15. Foucault M. A hermenêutica do sujeito. São Paulo: Martins Fontes; 2010.

16. Ferreira ML, Vargas MAO, Rodrigues J, Trentin D, Brehmer LCF, Lino MM. Comportamento Suicida e Atenção Primária à Saúde. Enfermagem em Foco do Cofen. 2018;9(4): 50-54. Disponivel em: http://revista.cofen.gov.br/index.php/ enfermagem/article/view/1803/477

17. Brasil. Pesquisa Nacional por Amostra de Domicílios - PNAD. 2020. Disponível em: https://www.ibge.gov.br/estatisticas/ sociais/trabalho/9171-pesquisa-nacional-por-amostra-de-domicilios-continua-mensal.html 
18. Gambarelli S, Taets G. A importância da empatia no cuidado de enfermagem na atenção primária à saúde. Enfermagem Brasil. 2018;17(4): 394-400. doi:10.33233/eb.v17i4.1258.

19. Unicovsky MAR, Santarem M. Desafios para enfermagem no atendimento aos pacientes idosos com deliruim e covid-19 em unidades de cuidados críticos. In: Enfermagem gerontológica no cuidado do idoso em tempo da COVID 19 / Associação Brasileira de Enfermagem. Brasília, DF: ABen/DCEG, 2020.

20. Conselho Federal de Enfermagem (COFEN). Resolução COFEN n 634 de 26 de março de 2020: autoriza e normatiza a teleconsulta de enfermagem como forma de combate à pandemia provocada pelo novo coronavírus (Sars-Cov-2). [Internet]. Brasília: COFEN; 2020 [acesso em 2020 abr 08]. Disponível em: http://www.cofen.gov.br/resolucaocofenno-0634-2020_78344.html 